\title{
Auswirkungen der Douglasie auf die Waldbiodiversität
}

\author{
Tobias Tschopp \\ Rolf Holderegger \\ Kurt Bollmann
}

Eidgenössische Forschungsanstalt für Wald, Schnee und Landschaft $(\mathrm{CH})$

Eidgenössische Forschungsanstalt für Wald, Schnee und Landschaft $(\mathrm{CH})^{*}$

Eidgenössische Forschungsanstalt für Wald, Schnee und Landschaft $(\mathrm{CH})$

\begin{abstract}
Auswirkungen der Douglasie auf die Waldbiodiversität
Unter dem Einfluss des Klimawandels propagiert die Waldwirtschaft in der Schweiz vermehrt den Anbau der exotischen Douglasie (Pseudotsuga menziesii), da diese Baumart trockenheitsresistenter als die einheimische Fichte ist. Demgegenüber befürchten Naturschutzorganisationen, dass der vermehrte Anbau der Douglasie sich negativ auf die Biodiversität auswirkt und dass die Douglasie das Potenzial zu invasiver Ausbreitung besitzt. Basierend auf der verfügbaren wissenschaftlichen und grauen Literatur stellen wir das heutige Wissen zu den Effekten der Douglasie auf Waldlebensräume und Biodiversität sowie zur Invasivität der Douglasie in Mitteleuropa zusammen. Der Anbau der Douglasie scheint im Vergleich mit einheimischen Nadelbäumen keine zusätzlichen negativen Auswirkungen auf den Boden zu haben und seine Effekte auf die Biodiversität sind sehr variabel und nicht konsistent. Grundsätzlich findet man in Douglasienbeständen eine Verschiebung der Artenzusammensetzung und der Dominanzverhältnisse bei den meisten der untersuchten Organismengruppen (z.B. bei Pilzen, Pflanzen, Arthropoden und Vögeln). Natürliche Verjüngung der Douglasie wurde in vielen Regionen Mitteleuropas gefunden. Deren Ausmass, Häufigkeit und Standortabhängigkeit und damit auch die Frage ihrer Invasivität sind jedoch noch nicht geklärt. Wir haben drei Wissenslücken identifiziert: 1) Das Verhalten der Douglasie entlang von Mischungsgradienten, insbesondere in Mischung mit der Buche, sollte untersucht werden, um jene Schwellenwerte im Mischungsgrad bestimmen zu können, ab denen negative Auswirkungen auf die Biodiversität auftreten. 2) Die Auswirkungen der Douglasie auf Arten der Roten Listen sowie auf national prioritäre oder charakteristische Waldarten müssen noch gründlich bestimmt werden. 3) Die Häufigkeit der natürlichen Verjüngung und das Ausbreitungspotenzial der Douglasie in Mitteleuropa sollten erfasst werden. Das Schliessen dieser Wissenslücken wird eine fachlich besser begründete und integrale Bewertung der Biodiversitätseffekte der Douglasie und ihres invasiven Potenzials erlauben.
\end{abstract}

Keywords: biodiversity, conservation biology, dispersal, Douglas fir, invasiveness, literature survey, soil doi: $10.3188 /$ szf. 2015.0009

*Zürcherstrasse 111, CH-8903 Birmensdorf, E-Mail rolf.holderegger@wsl.ch
$\mathrm{D}$ er Klimawandel stellt die Schweizer Waldwirtschaft vor neue Herausforderungen wie beispielsweise in Zukunft häufigere Trockenheit. Dabei wird oft auf die Douglasie (Pseudotsuga menziesii) verwiesen, die die trockenheitsanfällige Fichte ersetzen könnte. Die Vorteile der Douglasie sind ihre Wuchskraft, ein stabiles Wurzelwerk und ihre Toleranz gegenüber Sommertrockenheit (Höltermann et al 2008). Ihr Holz ist vielfältig einsetzbar (Sauter 1992).

Die Douglasie stammt aus dem westlichen Nordamerika, wo sie weit verbreitet ist. Es werden zwei Varietäten unterschieden: die Küstendouglasie (Pseudotsuga menziesii var. menziesii), die an der pazifischen Küste im Nordwesten von Nordamerika heimisch ist, und die Inlanddouglasie (Pseudotsuga menziesii var. glauca), welche in den Rocky Mountains von Mexiko bis ins südliche Kanada wächst (Aas 2008).
In der Schweiz wurde die Douglasie wahrscheinlich zum ersten Mal in Anbauversuchen des Schweizerischen Forstvereins im 19. Jahrhundert angepflanzt (Tschopp 2011). Heute ist sie in den tieferen Lagen der Schweiz, vor allem im Mittelland, zerstreut verbreitet. In den drei Landesforstinventaren wurde sie an insgesamt 83 Orten erfasst (Brändli 2010). Weitere Fundorte befinden sich im Jura, in den Voralpen, auf der Alpensüdseite und in den Zentralalpen (Bürgi \& Diez 1986, Datenbank des nationalen Daten- und Informationszentrums der Schweizer Flora ${ }^{1}$ ).

Rund die Hälfte der einheimischen Pilz-, Pflanzen- und Tierarten lebt mindestens teilweise im Wald (Duelli \& Coch 2004). Wegen dieser grossen Bedeutung des Waldes für die Biodiversität in der Schweiz stösst der in Zukunft möglicherweise ver-

1 www.infoflora.ch (25.11.2014) 
Abb 1 Anzahl berücksichtigter Publikationen nach Herkunftsland (aus Tschopp et al 2014). mehrte Anbau der Douglasie im Naturschutz auf Skepsis: Die Douglasie als nicht einheimische Baumart könnte die Biodiversität beeinträchtigen und sich invasiv verhalten (Eggert 2014a; zur Diskussion in Deutschland siehe z.B. Michl 2014). Der Anbau exotischer Baumarten ist aber auch mit waldbaulichen Risiken verbunden, wie zum Beispiel der Ausbreitung neuer Schädlinge und Pathogene (Sieber 2014). In der vorliegenden Arbeit wurde mittels Studium der wissenschaftlichen und der grauen Literatur untersucht, inwieweit der Anbau der Douglasie eine Gefährdung der Biodiversität in Mitteleuropa, insbesondere im Schweizer Wald, darstellt (für generelle Auswirkungen siehe Schmid et al 2014).

\section{Literaturstudie}

Es wurden Arbeiten aus Mitteleuropa (Belgien, Deutschland, Teilen von Frankreich, den Niederlanden, Österreich und Schweiz) berücksichtigt, da diese am ehesten auf die ökologischen Verhältnisse und die Biodiversität im Schweizer Wald übertragbar sind. Neben wissenschaftlichen Zeitschriften ${ }^{2}$ wurden auch Bücher, graue Literatur (z.B. unveröffentlichte Berichte und Studienarbeiten $)^{3}$ und Verweise auf weitere Untersuchungen in den aufgefundenen Publikationen in den jeweiligen Sprachen gesichtet. Wir fanden 92 relevante Untersuchungen

wichtigsten Schlussfolgerungen und stellen dann beispielhaft einige ausgewählte Studien vor, die diese Schlussfolgerungen illustrieren. Die vollständige und detaillierte Auswertung findet sich in der umfangreichen Studie von Tschopp et al (2014).

\section{Auswirkungen der Douglasie auf den Boden}

- Bestockungen mit Douglasie führen zur Versauerung und Nährstoffauswaschung im Oberboden. Inwieweit sich Bestockungen mit Douglasie dabei von solchen mit anderen Nadelbäumen unterscheiden, ist nicht klar. - Die Humusmächtigkeit unter Douglasien ist geringer als etwa unter Buchen und Föhren.

- Douglasienstreu gilt im Vergleich mit der Streu von anderen Nadelbaumarten als leicht zersetzbar.

Budde (2006) fand beim Vergleich von Buchen-, Föhren- und Douglasienbeständen in Niedersachsen für die Humusmächtigkeit einen signifikant tieferen Wert unter Douglasie. Die übrigen untersuchten Bodenparameter wie C/N-Verhältnis, $\mathrm{pH}$ Wert oder Stickstoffmineralisation lagen bei allen drei Baumarten eng beisammen. Mindrup et al (2001) verglichen die mikrobiologische Aktivität im Auflagehorizont von Douglasien- und Föhrenbeständen: Die Baumarten unterschieden sich in ihrem Einfluss auf die mikrobielle Aktivität nicht. Entscheidend waren vielmehr die standörtlichen Eigenschaften. Auch der Mischungsanteil der Douglasie wirkte sich nicht auf die mikrobielle Aktivität im Auflagehorizont aus. Burschel \& Huss (1997) erwähnten die leichte Zersetzbarkeit der Douglasienstreu, die alle andern in Deutschland angebauten Nadelbäume übertreffe.

\section{Auswirkungen der Douglasie auf die Flora}

- Die Bodenvegetation in Misch- und Reinbeständen der Douglasie ist ähnlich vielfältig wie in Laub- oder Nadelholzbeständen ähnlicher Standorte. Das Artenspektrum in Douglasienbeständen variiert allerdings stark. - Hinsichtlich des Vorkommens seltener, gefährdeter oder standorttypischer Arten in Douglasienbeständen ist wenig bekannt, und es können keine Schlussfolgerungen gezogen werden.

2014). Diese behandelten eines oder mehrere der Themengebiete Fauna, Naturverjüngung und Ausbreitung (Invasivität) sowie naturschutzfachliche Empfehlungen, hingegen weniger die Themengebiete Boden, Flora und Vegetation oder Pilze und Flechten. Die meisten Publikationen, nämlich 69, stammten aus Deutschland (Abbildung 1): 13 aus Baden-Württemberg und 18 aus Bayern.

Im Folgenden geben wir für jedes der oben genannten Themengebiete zuerst schlaglichtartig die
Kühnel (1995) verglich die Vegetation von Buchen- und Douglasienbeständen im Schwarzwald. An Nordhängen zeigten Buchen- im Vergleich zu Douglasienbeständen einen höheren Überschir-

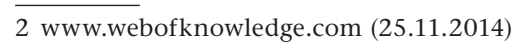

3 www.nebis.ch, www.bfw.ac.at, www.waldwissen.net (25.11.2014) 
mungsgrad, was die Lichtverhältnisse am Boden verschlechterte und einen tieferen Deckungsgrad der Strauch- und Krautschicht zur Folge hatte. Die Artenzahl in Douglasienbeständen war tendenziell geringer als in Buchenbeständen, und die Vegetation wurde von konkurrenzstarken Arten dominiert. Leitl (2001) verglich die Vegetation von Misch- und Reinbeständen von Fichte und Buche mit einem Douglasienreinbestand. Die meisten Pflanzenarten wurden im Fichtenreinbestand gefunden, gefolgt vom Douglasienreinbestand. Als Hauptursache wurde die Waldbewirtschaftung betrachtet, welche die Lichtverhältnisse im Fichtenreinbestand verbessert und verschiedene Kleinstandorte geschaffen hatte. Auch Augusto et al (2003) stellten fest, dass waldbauliche Eingriffe grossen Einfluss auf die Bodenvegetation hatten. Während nicht durchforstete Bestände mit Fichte, Tanne und Douglasie eine geringe Artenzahl aufwiesen, fanden sich in durchforsteten Beständen dieser Baumarten mehr Arten als in Laubholzbeständen. Leitl (2001) fand allerdings nur wenige Arten der natürlichen (Klimax-)Waldgesellschaften in einem Douglasienbestand, hingegen wies er viele Ruderal- und Schlagflurarten nach, welche naturschutzfachlich wenig Bedeutung hatten. Im Gegensatz dazu fanden Vor \& Schmidt (2006) viele Arten natürlicher Waldgesellschaften unter Douglasie.

\section{Auswirkungen der Douglasie auf Pilze und Flechten}

- Die Douglasie bildet in Mitteleuropa artenreiche Mykorrhiza-Gesellschaften aus, die jenen der Fichte und der Föhre gleichen.

- Die Artenvielfalt der Pilzflora in Douglasienbeständen ist tendenziell kleiner, und es werden mehr Generalisten als in naturnahen Waldbeständen nachgewiesen.

- Zu Auswirkungen der Douglasie auf Flechten in Mitteleuropa ist kaum etwas bekannt.

Le Tacon et al (1984) untersuchten die Entwicklung der Mykorrhiza in Pflanzungen von Fichte, Föhre und Douglasie. Die Artenzusammensetzung war allgemein ähnlich. Auch Pflanzungen auf Laubwaldstandorten entwickelten in kurzer Zeit Mykorrhiza, da ein Teil der Pilze, welche vorher mit Eiche, Buche oder Birke assoziiert waren, auch mit Douglasien, Fichten oder Föhren Symbiosen eingingen. Jansen (1991) untersuchte unterschiedlich alte Bestände der Douglasie und stellte in mehr als 20 Jahre alten Beständen weniger fruchtkörperbildende Mykorrhiza-Arten als in jüngeren Beständen fest. Utschick (2001) folgerte, dass Douglasienbestände im Vergleich mit Fichten- und Buchenbeständen beziehungsweise Naturwaldreservaten die geringsten Arten- und die geringsten Individuenzahlen an fruchtkörperbildenden Pilzen aufwiesen.
Römer (2001) verglich Anpflanzungen exotischer Nadelbäume, darunter der Douglasie, mit einem angrenzenden Kastanienwald im Tessin. Die Diversität der fruchtkörperbildenden Pilze im Kastanienwald war grösser als in den Pflanzungen gebietsfremder Baumarten. Die Mehrheit der Pilze unter Exoten waren Generalisten. Der hier aufgefundene, nicht einheimische Ektomykorrhiza-Pilz Rhizopogon villosulus wurde wohl mit der Douglasie aus Nordamerika eingeschleppt.

\section{Auswirkungen der Douglasie auf die Fauna}

- Es liegen viele Untersuchungen zu den Auswirkungen der Douglasie auf Vögel und verschiedene Arthropoden-Gruppen vor. Zu anderen Tiergruppen ist wenig bekannt.

- Bei den Arthropoden sind die Resultate abhängig von der Artengruppe, der Baumartenmischung und der Jahreszeit: Häufigkeit und Anzahl sind bei gewissen Artengruppen auf Douglasie grösser (z.B. bei Läusen), während sie für andere im Vergleich zu einheimischen Baumarten kleiner sind (z.B. bei Spinnen). Im Stammbereich der Douglasie werden, vor allem im Winter, weniger Arten und Individuen als auf Fichte festgestellt.

- Insgesamt kommt es in Douglasienbeständen im Vergleich zu naturnahen Beständen bei den Arthropoden zu einer Verschiebung des Artenspektrums und der dominanten Arten.

- Im Vergleich zu einheimischen Baumarten wird auf Douglasien ein Rückgang der Häufigkeit und Diversität von Vögeln festgestellt. Im Vergleich mit einheimischen Nadelbaumbeständen weisen ältere Douglasienbestände jedoch eine ähnlich grosse oder sogar grössere Häufigkeit und Artenvielfalt von Vögeln auf.

Lebreton \& Pont (1987) fanden im Vergleich mit Fichten- und Weisstannenbeständen die geringsten Artenzahlen und Häufigkeiten von Vögeln in Douglasienbeständen. Im Gegensatz dazu zeigte Utschick (2001), dass sich in Douglasienbeständen mehr Vogelarten als in Fichtenbeständen, aber weniger als in Mischbeständen aus Fichten und Buchen, reinen Laubholzbeständen oder Naturwaldreservaten finden lassen. Müller et al (1994) kartierten nur 55 bis 60 Prozent der Anzahl Brutvogelpaare pro Hektare in einem Douglasienbestand verglichen mit zwei Fichtenbeständen.

Gossner \& Utschick (2004) zeigten, dass die Vogelaktivität in Douglasienreinbeständen im Vergleich zu verschiedenen anderen Beständen über das ganze Jahr gesehen am niedrigsten war. Vor allem im Winter wurde kaum Vogelaktivität beobachtet. Als Hauptursache wurde ein vermindertes Nahrungsangebot an Arthropoden auf Douglasie angenommen. In Mischbeständen aus Fichte und Douglasie war hingegen kein negativer Effekt betreffend Vogelaktivität auszumachen. 


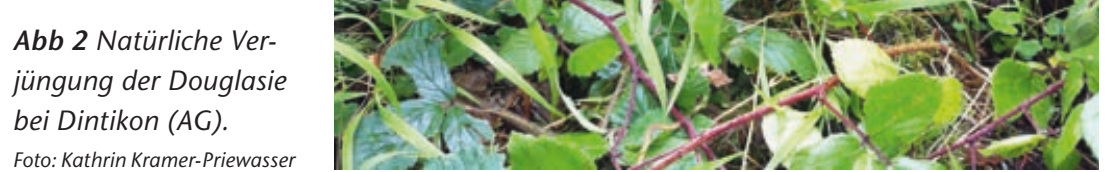

Für Arthropoden liegt eine Vielzahl von Untersuchungen mit allerdings oft inkonsistenten Ergebnissen vor. Kilchling (1993) fand keine Unterschiede in der Individuenzahl von Arthropoden zwischen Douglasie und Weisstanne. Allerdings gab es Verschiebungen im Artenspektrum und in den dominanten Arten. Auf Douglasie wurden zudem viele kleine, wenig spezialisierte Insekten gefunden. Keine Unterschiede wurden auch in der Käferfauna eines Föhren-Buchen- und eines Douglasien-BuchenMischbestandes gefunden (Glatz et al 2003). Blick \& Gossner (2006) fanden für Spinnen fast gleiche Artenzahlen auf Fichte und Douglasie. In einer kürzlich veröffentlichten Studie wurde ebenfalls ein negativer Effekt der Douglasie auf die Spinnenfauna im Vergleich zu Buche, Traubeneiche und Fichte in experimentellen Pflanzungen dieser Baumarten ermittelt (Schuldt \& Scherer-Lorenzen 2014). Auch Kohlert \& Roth (2000) fingen in einem Fichtenbestand fast dreimal so viele Individuen von Spinnen wie in einem Douglasienbestand. Zusammenfassend schlossen Winter et al (2001), dass es sowohl in Reinals auch in Mischbeständen der Douglasie zuerst zu einer Verarmung der Arthropodenfauna kommt, sich der Arthropodenbestand dann allerdings langfristig an jenen einheimischer Nadelholzbestände angleicht. Hingegen sind Ammer \& Utschick (2004) der Meinung, dass grossflächiger Douglasienanbau eine reduzierte Artenvielfalt und einen erhöhten Anteil an Generalisten unter den Arthropoden zur Folge hat.

\section{Invasivität der Douglasie}

- Praktisch in allen untersuchten Gebieten in Mitteleuropa ist Naturverjüngung der Douglasie beobachtbar. - Douglasien-Naturverjüngung findet sich auf trockenen, mageren und eher sauren Standorten. Hier ist sie konkurrenzstark und scheint andere Baumarten zu verdrängen.

- Die Douglasie fruktifiziert früh, im Alter von 30 bis 50 Jahren, und ihre Samen verbreiten sich nachweislich bis zu 200 Meter weit.

Guntermann (1989) fand in 47 Prozent seiner Probeflächen im Schwarzwald Douglasienverjüngung (Abbildung 2), wobei die Individuenzahl stark variierte. Bei Überschirmungsgraden von über 80 Prozent fand sich keine Douglasienverjüngung. Falk (1988) zeigte, dass Douglasienverjüngung südexponierte, relativ trockene und unfruchtbare Böden bevorzugt. Als Grund wurde die geringere Konkurrenz auf solchen Standorten angegeben. Kühnel (1995) zeigte, dass im Schwarzwald Douglasienverjüngung an Nordhängen von der Buche dominiert wird, auf Südhängen verhielt es sich jedoch genau umgekehrt. Nach Annen (1998) können auf nährstoffarmen oder sandigen Standorten beachtliche Jungwuchsdichten der Douglasie von über 25000 Individuen pro Hektare auftreten. Zudem keimt die Douglasie gerne auf gestörten Oberböden.

Die Douglasie fruktifiziert früh, im Alter von 30 bis 50 Jahren, und Verjüngung tritt in einem Umkreis von 200 Metern um die Samenbäume auf (Appelfelder 1999, Eggert 2014a). Knoerzer (2004) modellierte hingegen Ausbreitungsdistanzen von Douglasiensamen von bis zu 380 Metern. Genetische Untersuchungen zur Ausbreitung der Douglasie im Mitteleuropa liegen nicht vor.

\section{Naturschutzfachliche Bewertung der Douglasie in der Literatur}

- Grundsätzlich spricht sich kein Autor gegen Douglasienanbau aus. Allerdings besteht Übereinstimmung darüber, dass dies ausschliesslich in Mischung, zum Beispiel mit Buche, erfolgen sollte.

- Douglasienanbau soll auf gut mit Wasser und Nährstoffen versorgte Böden beschränkt werden, wo die Verjüngung der Douglasie besser kontrolliert werden kann. Auf trockenen und nährstoffarmen Standorten wird die Ausbreitung der Douglasie befürchtet.

- Allgemein wird in der Literatur vorgeschlagen, dass die Douglasie auf für sie passenden, jedoch schützenswerten Standorten und in Naturvorranggebieten nicht angebaut werden soll.

Knoerzer (1998) und andere Autoren zählen die Douglasie zu den absichtlich eingeführten Neophyten. Schwenk \& Pauli (2012) fanden in einer Befra- 
«quasi-experimenteller» Ansatz verfolgt werden, mit welchem die Effekte der Douglasie entlang von Mischungsgradienten, bestehend aus bereits existierenden Beständen mit verschiedenen Mischungsanteilen der Douglasie, untersucht werden. So könnten jene Schwellenwerte bestimmt werden, ab denen nachweislich negative Effekte der Douglasie auf die Waldbiodiversität auftreten. Auf diese Weise liesse sich auch die Frage des «Entweder-oder»-Referenzsystems umgehen (z.B. Douglasie gegenüber Buche), sondern es würde ein Gradient (z.B. Douglasie in verschiedener Mischung mit Buche) untersucht.

- Eine naturschutzfachliche Bewertung der Effekte der Douglasie auf charakteristische Waldarten, Rote-Liste-Arten oder national prioritäre Arten auf verschiedenen Standorten steht für die Schweiz noch aus.

- Dass sich die Douglasie auf geeigneten Standorten natürlich verjüngt, steht ausser Frage. Offen ist hingegen, in welchem Ausmass und in welchen Waldgesellschaften des Schweizer Klassifizierungssystems sie das tut. Zudem ist unbekannt, wie häufig diese Waldgesellschaften oder die entsprechenden Standorte in Zukunft unter dem Klimawandel sein werden. - Das Ausbreitungspotenzial der Douglasie ist noch nicht genügend erforscht. Insbesondere muss ihr Fernausbreitungsvermögen bestimmt werden, da dieses für die Invasivität einer Art entscheidend ist.

Erst wenn bekannt ist, welche Effekte die Douglasie in welchem Mischungsverhältnis auf welchen Standorten auf Waldlebensräume hat, wie weit sie sich ausbreiten kann und wo sie sich in welchem Ausmass natürlich verjüngt, können die Biodiversitätseffekte und die Invasivität der Douglasie in der regional reich strukturierten Schweiz besser abgeschätzt werden.

Eingereicht: 26. September 2014, akzeptiert (mit Review): 19. November 2014

\section{Dank}

Wir danken dem Bundesamt für Umwelt für die Finanzierung der Studie.

\section{Literatur}

AAS G (2008) Die Douglasie (Pseudotsuga menziesii) in Nordamerika: Verbreitung, Variabilität und Ökologie. Freising: Bayer Landesanstalt Wald Forstwirtschaft, LWF Wissen 59. pp. 7-11. AMMER U, UTSCHICK H (2004) Folgerungen aus waldökologischen Untersuchungen auf hochproduktiven, nadelholzreichen Standorten für eine an Naturschutzzielen orientierte Waldwirtschaft. Forst Holz 59: 119-128.

ANNEN H (1998) Zum Einfluss von Oberbodenzustand und Standort auf Samenkeimung und Verjüngungsdichte der Douglasie in Südwestdeutschland. Freiburg i.Br.: Albert-LudwigsUniv, Dissertation. 160 p.

APPELFELDER J (1999) Anmerkungen zur Konkurrenzsituation der Naturverjüngung und zur Ausbreitung der Douglasie (Pseu- dotsuga menziesii) im Naturschutzgebiet Lüneburger Heide. Göttingen: Georg-August-Univ, Diplomarbeit. 92 p.

AUGUSTO L, DUPOUEY JL, RANGER J (2003) Effects of tree species on understory vegetation and environmental conditions in temperate forests. Ann Sci For 60: 823-831.

BLICK T, GOSSNER M (2006) Spinnen aus Baumkronen-Klopfproben (Arachnida: Araneae) mit Anmerkungen zu Cinetata gradata (Linyphiidae) und Theridion boesenbergi (Theridiidae). Arachnol Mitt 31: 23-39.

BRÄNDLI UB (2010) Schweizerisches Landesforstinventar. Ergebnisse der dritten Erhebung 2004-2006. Birmensdorf: Eidgenöss Forsch.anstalt WSL. 312 p.

BUDDE S (2006) Auswirkungen des Douglasienanbaus auf die Bodenvegetation im nordwestdeutschen Tiefland. Göttingen: Georg-August Univ, Dissertation. 111 p.

BÜRGI A, DIEZ C (1986) Übersicht über den Exotenanbau in der Schweiz aufgrund einer Umfrage vom Herbst/Winter 1984/85. Schweiz Z Forstwes 137: 833-851.

BURSCHEL P, HUSS J (1997) Grundriss des Waldbaus. Berlin: Parey. $487 \mathrm{p}$.

DUELLI P, COCH T (2004) Biodiversität im Schweizer Wald. Bern: Forum Biodiversität, Hotspot 9: 6-7.

EGGERT M (2014A) Wie verjüngt sich die Douglasie? Allg Forst Z Waldwirtsch Umweltvorsorge 96 (11): 27-29.

EGGERT M (2014B) Ökologische und waldbauliche Aspekte des Douglasienanbaus. Allg Forst Z Waldwirtsch Umweltvorsorge 96 (11): 31-32.

FALK B (1988) Ein Beitrag zur Naturverjüngung der Douglasie. Freiburg i.Br.: Albert-Ludwigs-Univ, Diplomarbeit. 69 p.

GLATZ K, WINTER K, NIEMEYER H (2003) Beitrag zur epigäischen Käferfauna in niedersächsischen Mischwäldern mit und ohne Douglasie. Forst Holz 58: 32-36.

GOSSNER M, UTSCHICK H (2004) Douglas fir stands deprive wintering bird species of food resource. Neobiota 3: 105-122.

GUNTERMANN T (1989) Untersuchungen zur natürlichen Verjüngung der Douglasie im Stadtwald Sulzburg. Freiburg i.Br.: Albert-Ludwigs-Univ, Diplomarbeit. 111 p.

HÖLTERMANN A, KLINGENSTEIN F, SSYMANK A (2008) Naturschutzfachliche Bewertung der Douglasie aus Sicht des Bundesamtes für Naturschutz (BfN). Freising: Bayer Landesanstalt Wald Forstwirtschaft, LWF Wissen 59. pp. 74-81.

JANSEN AE (1991) The mycorrhizal status of Douglas fir in The Netherlands: its relation with stand age, regional factors, atmospheric pollutants and tree vitality. Agric Ecosyst Environ 35: 191-208.

KILCHLING K (1993) Die tierökologische Bedeutung der Stammregion der fremdländischen Baumarten Roteiche und Douglasie im Vergleich zu Stieleiche und Fichte/Tanne. Freiburg i.Br.: Albert-Ludwigs-Univ, Diplomarbeit. 140 p.

KNOERZER D (1998) Zum Status nichtheimischer (Baum-)Arten von der Notwendigkeit begrifflicher Klärung. Allg ForstJagdztg 169: 41-46.

KNOERZER D (1999) Zur Naturverjüngung der Douglasie im Schwarzwald. Inventur und Analyse von Umwelt- und Konkurrenzfaktoren sowie eine naturschutzfachliche Bewertung. Berlin: Borntraeger. 283 p.

KNOERZER D (2004) The dispersal of Douglas-fir diaspores in forests in Germany. Neobiota 3: 55.

KOHLERT A, ROTH M (2000) Der Einfluss fremdländischer Baumarten (Douglasie: Pseudotsuga menziesii) auf saprophage Arthropoden und epigäische Regulatoren. Mitt Dtsch Ges allg angew Entom 21: 71-74.

KOWNATZKI D, KRIEBITZSCH WU, BOLTE A, LIESEBACH H, SCHMITT U, ELSASSER P (2011) Zum Douglasienanbau in Deutschland ökologische, waldbauliche, genetische und holzbiologische Gesichtspunkte des Douglasienanbaus in Deutschland und den angrenzenden Staaten aus naturwissenschaftlicher und gesellschaftspolitischer Sicht. Braunschweig: Bundesforschungsinstitut Ländliche Räume Wald Fischerei. 78 p. 
KÜHNEL U (1995) Zum Einfluss des Douglasienanbaus auf buchendominierte Waldökosysteme. Freiburg i.Br.: Albert-Ludwigs-Univ, Diplomarbeit. $88 \mathrm{p}$.

LE TACON F, LAMOURE D, GUIMBERTEAU J, FIKET C (1984) Les symbiotes mycorhiziens de l'épicéa commun et du douglas dans le Limousin. Rev for fr 36: 325-328.

LEBRETON P, PONT B (1987) Avifaune et altérations forestières. I. L'avifaune des boisements résineux du Haut-Beaujolais: considérations générales. Acta Oecol 8: 227-235.

LEITL R (2001) Artenvielfalt und Bestandesform am Beispiel der Bodenvegetation. Freising: Bayer Landesanstalt Wald Forstwirtschaft, LWF Wissen 33. pp. 9-13.

MEYER P (2011) Naturschutzfachliche Bewertung der Douglasie. Forstarchiv 82: 157-158.

MICHL A (2014) Invasive Art - was heisst das konkret? Allg Forst Z Waldwirtsch Umweltvorsorge 96 (11): 33-34.

MINDRUP M, MEIWES KJ, WOLTERS V (2001) Mikrobiologische Eigenschaften des Auflagehumus unter Douglasie und Kiefer in Nordwestdeutschland. Forst Holz 56: 363-366.

MÜLLER J, STOLLENMEIER H, STOLLENMEIER S (1994) Auswirkungen des Douglasienanbaus auf die Vogelwelt. Allg Forst Z Waldwirtsch Umweltvorsorge 86 (5): 237-239.

RÖMER N (2001) Einfluss von Aufforstungen mit standortsfremden Nadelbaumarten auf die Pilzflora im Laubwaldgürtel in der Südschweiz (TI, S. Antonino, Copera). Zürich: ETH Zürich, Dissertation. 208 p.

SAUTER UH (1992) Die Douglasie - eine Holzart mit Potential. Freiburg i.Br.: Forstl Vers- Forsch.anstalt Baden-Württemberg, FVA-Einblick 14 (3). pp. 13-14.

\section{Impact du sapin douglas sur la biodiversité forestière}

Face au changement climatique, l'économie forestière suisse promeut le recours au sapin douglas en sylviculture. Le douglas (Pseudotsuga menziesii), espèce non autochtone, supporte en effet mieux la sécheresse que l'épicéa indigène. Cette pratique inquiète les organisations de protection de la nature: elles craignent qu'une plantation généralisée du douglas ait un impact négatif sur la biodiversité et que cette essence forestière se révèle invasive. Les connaissances actuelles de l'impact du douglas sur les habitats forestiers et sur la biodiversité, tout comme son potentiel invasif en Europe centrale sont présentés ici, en faisant référence aux publications scientifiques et générales disponibles sur le sujet. La plantation de douglas ne semble pas avoir plus d'effets négatifs sur le sol que celle de conifères autochtones. Ses effets sur la biodiversité sont très divers et inconsistants. En principe, on trouve dans les peuplements de douglas un changement dans la composition des espèces et dans les relations de dominance avec la plupart des groupes d'organismes étudiés (p.ex. champignons, plantes, arthropodes et oiseaux). Une régénération naturelle du douglas est observée dans plusieurs régions d'Europe centrale, mais son ampleur, son abondance, l'influence de la station et donc le potentiel invasif du douglas ne sont pas encore clarifiés. D'après notre étude, il reste à élucider les points suivants: 1) II faudrait mener des études concernant le degré de mélange avec d'autres essences, en particulier avec le hêtre, afin de définir le seuil de la proportion de douglas préjudiciable à la biodiversité. 2) L'impact du douglas sur les espèces Liste Rouge, les espèces prioritaires et caractéristiques de la forêt reste à identifier. 3) Le potentiel de propagation et de rajeunissement naturel du douglas en Europe centrale doivent encore être étudiés. La réponse à ces questions permettra une évaluation scientifiquement plus étayée et plus intégrale des effets du douglas sur la biodiversité et sur son potentiel invasif.
SCHMID M, PAUTAUSSO M, HOLDENRIEDER O (2014) Ecological consequences of Douglas fir (Pseudotsuga menziesii) cultivation in Europe. Eur J For Res 133: 13-29.

SCHULDT A, SCHERER-LORENZEN M (2014) Non-native tree species (Pseudotsuga menziesii) strongly decreases predator biomass and abundance in mixed-species plantations of a tree diversity experiment. For Ecol Manage 327: 10-17.

SCHWENK U, PAULI B (2012) Chancen und Risiken. Wald Holz 93 (4): 37-39.

SIEBER TN (2014) Neomyzeten - eine anhaltende Bedrohung für den Schweizer Wald. Schweiz Z Forstwes 165: 173-182. doi: 10.3188/szf.2014.0173

TSCHOPP T (2011) Geschichte der exotischen Baumarten in der Schweiz. Zürich: ETH Zürich, Masterarbeit. 73 p.

TSCHOPP T, HOLDEREGGER R, BOLLMANN K (2014) Die Douglasie in der Schweiz: Auswirkungen auf Biodiversität und Lebensräume im Wald. Birmensdorf: Eidgenöss Forsch.anstalt WSL, WSL Ber 20. 52 p.

UTSCHICK H (2001) Vögel, Schnecken, Pilze. Freising: Bayer Landesanstalt Wald Forstwirtschaft, LWF Ber 33. pp. 45-49.

VOR T, SCHMIDT W (2006) Auswirkungen des Douglasienanbaus auf die Vegetation der Naturwaldreservate «Eselskopf» (Nordwesteifel) und «Grünberg» (Pfälzer Wald). Forstarchiv 77: 169-178.

WINTER K, FINCH OD, GLATZ K (2001) Zur Arthropodenfauna in niedersächsischen Douglasienforsten: II. Mischbestände im Flachland. Forst Holz 56: 720-726.

\section{Effects of Douglas fir on forest biodiversity}

Under climate change, forestry in Switzerland promotes the increased cultivation of exotic Douglas fir (Pseudotsuga menziesii), as Douglas fir is more drought-resistant than native spruce. However, nature conservation organisations fear that enhanced planting of Douglas fir will negatively affect biodiversity and that Douglas fir has invasive potential. Based on the existing scientific and grey literature, we compile the present knowledge on the effects on forest habitats and biodiversity and the invasiveness of Douglas fir in Central Europe. The cultivation of Douglas fir does not seem to have any additional negative effects on soils as compared to native conifers, and its effects on biodiversity are diverse and often inconsistent. Overall, there is often a shift in species composition and in the dominance ratio for most studied groups of organisms (e.g. fungi, plants, arthropods, birds) in Douglas fir stands. Although natural regeneration of Douglas fir does occur in many regions of Central Europe, its extent, site-specificity and frequency and, therefore, the invasiveness of Douglas fir are not yet clear. We identified the following knowledge gaps: 1) Douglas fir should be studied along mixture gradients with other tree species, especially beech, in order to determine threshold values at which negative effects of biodiversity begin to appear. 2) The effects of Douglas fir on Red List, priority or characteristic forest species have not yet been thoroughly evaluated. 3) Frequency of natural regeneration and dispersal potential of Douglas fir in Central Europe should be assessed. Filling these knowledge gaps will allow a more reliable and integral assessment of the biodiversity effects of Douglas fir and its invasive potential. 